APJ LETTERS IN PRESS

Preprint typeset using $\mathrm{LAT}_{\mathrm{E} X}$ style AASTeX6 v. 1.0

\title{
CHROMOSPHERIC MODELS AND THE OXYGEN ABUNDANCE IN GIANT STARS
}

\author{
A. K. Dupree, E. H. Avrett, and R. L. Kurucz \\ Harvard-Smithsonian Center for Astrophysics, Cambridge, MA 02138, USA
}

\begin{abstract}
Realistic stellar atmospheric models of two typical metal-poor giant stars in Omega Centauri that include a chromosphere (CHR) influence the formation of optical lines of O I: the forbidden lines $(\lambda 6300$, $\lambda 6363)$ and the infrared triplet $(\lambda \lambda 7771-7775)$. One-dimensional semi-empirical non-LTE models are constructed based on observed Balmer lines. A full non-LTE formulation is applied in evaluating line strengths of $\mathrm{O}$ I including photoionization by the Lyman continuum and photoexcitation by Ly- $\alpha$ and Ly- $\beta$. Chromospheric models (CHR) yield forbidden oxygen transitions that are stronger than in radiative/convective equilibrium (RCE) models. The triplet oxygen lines from high levels also appear stronger than produced in an RCE model. The inferred oxygen abundance from realistic CHR models for these two stars is decreased by factors $\sim 3$ as compared to values derived from RCE models. A lower oxygen abundance suggests that intermediate mass AGB stars contribute to the observed abundance pattern in globular clusters. A change in the oxygen abundance of metal-poor field giants could affect models of deep mixing episodes on the red giant branch. Changes in the oxygen abundance can impact other abundance determinations critical to astrophysics including chemical tagging techniques and galactic chemical evolution.
\end{abstract}

Keywords: stars: atmospheres — stars: abundances — stars: chromospheres — line: formation

\section{INTRODUCTION}

Determination of stellar abundances constitutes a fundamental component of a wide variety of issues in contemporary astrophysics. Large spectroscopic surveys of chemical compositions can reveal the history of star formation in our Galaxy and the early universe (Frebel \& Norris 2015). Ages of clusters are inferred from chemical abundances ( Fulbright \& Johnson 2003; Maderak et al. 2015; Marino et al. 2012a, 2012b). The origin of multiple populations in globular clusters is addressed by abundances and remains not understood (Gratton et al. 2012, Renzini et al. 2015).

The $\mathrm{O}$ I atom produces two sets of transitions in stars that are frequently used to evaluate the oxygen abundance: the forbidden $[\mathrm{O} \mathrm{I}]$ lines at $6300 \AA$ and $6363 \AA$ and the $\mathrm{O}$ I triplet at $7771-7775 \AA$. Abundances inferred using radiative/convective equilibrium (RCE) models yield values in giant stars that can differ by 0.3 to 1.2 dex depending on the oxygen transition (Fulbright \& Johnson 2003; Takeda 2003; Israelian et al. 2004). While it has been asserted that the forbidden transitions appear to be in local thermodynamic equilibrium (LTE) using photospheric models (Kiselman 2001; Takeda 2003; Asplund 2005), non-LTE effects can create an apparently stronger triplet transition in a RCE model (Takeda 2003, Shchukina et al. 2005; Schuler et al. 2006; Fabbian et al. 2009; Amarsi et al. 2015) requiring a slightly lower oxygen abundance. Takeda (2003) noted that even taking into account non-LTE corrections in a RCE model, metal-poor disk/halo stars still exhibit systematic anomalies between diagnostics that can amount to a median value of $\sim 0.3 \mathrm{dex}$. Authors have conjectured that these discrepancies arise from inadequate model atmospheres or in the calculation of spectral lines (Israelian et al. 2004; Shchukina et al. 2005; Schuler et al. 2006).

Critical to the determination of abundances are the stellar models used to extract abundance values. Stellar models (i.e. MARCS, PHOENIX, ATLAS) traditionally consist of a one-dimensional radiative and convective equilibrium (RCE) stellar photosphere in hydrostatic equilibrium. The temperature decreases from the interior of the star outwards with constant radiative and convective flux and with gravity balancing the pressure gradient (Gustafsson et al. 2008; Castelli \& Kurucz 2004; Hauschildt et al. 1999; Mészáros et al. 2012). The level populations and line strengths are computed in LTE. In some instances, adjustments are made to construct so-called non-LTE corrections to the RCE

dupree@cfa.harvard.edu 
models for O I (Takeda 1997; Gratton et al. 1999), usually with simplifying assumptions.

Another development includes the construction of three-dimensional models of a stellar photosphere with hydrodynamical simulations of radiative and convective transport (Asplund et al. 1999; Freytag et al. 2010). Such models have been used (cf. Dobrovolskas et al. 2014) to assess the impact of inhomogeneities in the photospheres in cool stars and the appropriate correction factors for abundances when derived from a 1-D model.

However, these previous models for giant stars are unrealistic because chromospheres are missing. Many if not all cool stars exhibit spectroscopic signs of non-radiative heating which creates chromospheres with temperatures in excess of the photospheric temperature. Optical stellar spectra signal the presence of chromospheres in a variety of ways: emission in the cores of the $\mathrm{Ca}$ II $\mathrm{H}$ and $\mathrm{K}$ lines and $\mathrm{Na}$; emission in the wings of $\mathrm{H} \alpha$; and the presence of near-ir He I (Dupree et al. 1984; Dupree et al. 2007; Dupree \& Smith 1995; Mészaros et al. 2009; Martínez-Arnáiz et al. 2010).

In this paper, we make the first calculations of optical line profiles for oxygen in two giant stars using a realistic atmospheric model that includes a chromosphere (CHR). We chose the O I atom because optical transitions in oxygen are widely used in stellar abundance determinations of cool luminous stars (Boesgaard et al. 2015, Korotin et al. 2014, VandenBerg et al. 2014; Johnson et al. 2013; Johnson \& Pilachowski 2010, 2012; Gratton et al. 2015). Oxygen, the third most abundant element, remains pivotal in the astronomical context. Some have suggested that oxygen is superior to iron as a tracer of galactic chemical evolution because the sites for its synthesis are better understood (Wheeler et al. 1989).

\section{THE CALCULATION STRATEGY}

Oxygen level populations are calculated assuming a radiative/convective equilibrium (RCE) model in LTE to provide comparison profiles. Then an atmospheric model is constructed with a chromosphere (CHR) to match the observed $\mathrm{H} \alpha$ and $\mathrm{H} \beta$ line profiles of two red giants in Omega Centauri. Because these Balmer lines are produced in a wide region of the chromosphere, they link the photospheric model to that of the chromosphere and define a reliable semiempirical model. This non-LTE model based on hydrogen is used to calculate the oxygen populations and the line profiles, treating oxygen as a trace element. The effects of the Lyman continuum and the Ly- $\alpha$ and Ly- $\beta$ line (Bowen fluorescence), calculated in full non-LTE approximation, are included in the ionization and excitation of oxygen.

\section{THE PANDORA CODE}

We use the PANDORA code (Avrett \& Loeser 2008) to construct a one-dimensional semi-empirical atmosphere by iteratively evaluating for the hydrogen atom, the ionization state, line and continuum radiation, and level populations. The atmospheric structure ( $T, N_{\mathrm{H}}$, and turbulent broadening) is modified until the calculated line profiles agree with observation. The value of $N_{\mathrm{H}}$ is determined empirically rather than by hydrostatic equilibrium. The total model is iterated with full non-LTE calculations in order to match the specified line profiles. All transitions are calculated explicitly. Calculations are carried out in plane-parallel geometry, evaluating the profile at various values of $\mu=\cos \theta$, and then integrating over all angles.

\section{THE ATOMIC MODELS AND RATES}

The atomic model for H I replicates that used in Avrett \& Loeser (2008) with a 15-level H atom plus continuum that involves 105 line transitions. Partial frequency redistribution is used for the calculation of the Ly- $\alpha$ and Ly- $\beta$ profiles. The Lyman continuum is also included as it affects the hydrogen ionization. A calculation for hydrogen is made first in order to determine the ionization structure in the atmosphere by iterating to fit the observed $\mathrm{H} \alpha$ and $\mathrm{H} \beta$ profiles. The Lyman series profiles are evaluated explicitly. Once the ionization structure and the electron density are known, then level populations and line profiles for trace atoms such as O I, can be calculated.

The atomic model for O I consists of 40 individual energy levels grouped into 20 reference levels. This includes the ground level of O II and two levels in the continuum of O I. Electron and hydrogen collisions between all levels are included, as is collisional ionization by electrons and hydrogen and photoionization from all levels including effects of the Lyman continuum. Radiative and dielectronic recombination are included. Electron-impact excitation rates are taken from calculations of Barklem (2007), and supplemented by those of Seaton (1962). Hydrogen-impact rates are from Kaulakys (1985) and Drawin (1969). Dielectronic recombination rates are taken from Badnell et al. (2003) and their fitting parameters were employed.

Of particular import for neutral oxygen is the Bowen mechanism (Bowen 1947) in which the coincidence in wavelength between Ly- $\beta$ and the O I transition from the ground state ${ }^{3} \mathrm{P}$ to an excited ${ }^{3} \mathrm{D}$ level $\left(2 p^{4}{ }^{3} P-2 p^{3} 3 d^{3} D\right)$ increases the populations in the triplet sequence which also contributes to the intersystem collision rates. The strength of the O I emission in the first ultraviolet spectrum of a giant star, $\alpha$ Boo, was found to be surprisingly strong (Moos \& Rottman 
1972) and was subsequently understood to result from Ly- $\beta$ pumping as proposed by Bowen (Haisch et al. 1977). UV surveys document the dominating strength of the O I resonance lines in cool luminous stars (Ayres et al. 1995) and require inclusion of photoexcitation and photoionization from chromospheric hydrogen radiation in the calculation of oxygen level populations and ionization.

\section{ATMOSPHERIC MODEL}

A model was constructed loosely based on two giant stars in the globular cluster Omega Centauri. We select a model with $T_{\text {eff }}=4745 \mathrm{~K}, \log g=1.74,[\mathrm{Fe} / \mathrm{H}]=-1.7$, and $\alpha$-enhancement $([\alpha / \mathrm{Fe}]=+0.4)$. These parameters are consistent with spectroscopic analyses of LEID 54084 and LEID 54064 in Omega Centauri (Johnson \& Pilachowski 2010; Dupree et al. 2011). Solar abundances (Asplund et al. 2009) are decreased by -1.7 dex and the alpha elements ( $\mathrm{Ne}, \mathrm{Mg}, \mathrm{Si}, \mathrm{S}, \mathrm{Ar}, \mathrm{Ca}$, and $\mathrm{Ti}$ ) are enhanced by +0.4 dex relative to the scaled solar values. The abundance of oxygen is first taken as $\log \mathrm{O}=7.64$ where $\log \mathrm{H}=12.00$; subsequently this abundance is changed in additional calculations. A radiative/convective equilibrium (RCE) and a chromospheric model (CHR) were constructed. The temperature distributions for these models are shown in Figure 1 and discussed below.

\subsection{Radiative/Convective Equilibrium Model (RCE)}

This model in hydrostatic equilibrium exhibits a monotonically decreasing temperature with height. The Kurucz model (Castelli and Kurucz 2004) was calculated specifically for the stellar parameters listed above. Values of turbulent broadening are introduced to match the observed width of the Balmer lines in the stars. The RCE model does not produce good fits to the Balmer line profiles. It has long been known (Dupree et al. 1984) that the RCE approximation is not expected to match the observed profiles because Balmer lines have a chromospheric component.

\subsection{Chromospheric Models (CHR)}

The construction of a semi-empirical chromospheric model (CHR) relies on matching appropriate line profiles. The $\mathrm{H} \alpha$ and $\mathrm{H} \beta$ lines are useful in this regard since the atmospheric levels of profile formation span a large temperature region in low gravity stars (Mészaros et al. 2009). The RCE model is extended in height to $1.8 \mathrm{R}_{\star}$ (where $\mathrm{R}_{\star}=30 \mathrm{R}_{\odot}$ ) along with a temperature increasing to $10^{5} \mathrm{~K}$ with height. We solve the non-LTE optically thick transfer equation for hydrogen and modify the atmospheric model (Fig. 1) to produce good agreement with the $\mathrm{H} \alpha$ and $\mathrm{H} \beta$ line profiles (Fig. 2 ). These profiles originate from the high photosphere in the wings of the line, traverse the temperature minimum, and arise from the low chromosphere in the line core as shown in Fig. 1. We take this semi-empirical model as the basis for the oxygen calculations. The Ly- $\beta$ profile from the hydrogen model is used as the input for photoexcitation of the ${ }^{3} \mathrm{D}$ level of O I. The Lyman continuum and Ly- $\alpha$ from the hydrogen model are also included to evaluate the oxygen ionization and excitation.

A recent calculation of oxygen in metal-poor stars using a 3D RCE model (Amarsi et al. 2016) notes the importance of the Ly- $\alpha$ wings in photoexcitation of the oxygen resonance lines. A RCE model, lacking a chromosphere will not produce an accurate Lyman continuum value nor a realistic Ly- $\alpha$ profile, so its influence is difficult to assess. In addition, the Ly- $\alpha$ profile needs to be calculated with partial frequency distribution which will lower the flux in the line wings. Unfortunately, the Ly- $\alpha$ profile is challenging to measure directly because of interstellar absorption and geocoronal emission.

\section{OXYGEN LINE PROFILES}

Line profiles for three oxygen transitions are shown in Figure 3. The CHR solutions are stronger than the RCE/LTE profiles for all transitions. The forbidden transitions reach optical depth unity deep in the atmosphere where higher densities and greater collisional coupling occurs. However, the levels are not in their LTE population ratios where the forbidden lines are formed (Fig. 1). The strengthening of the triplet transition has been noted previously in RCE models by a number of authors (Takeda 2003, Fabbian et al. 2009), but the chromospheric model strengthens the lines to a greater degree.

To evaluate the sensitivity of the line strength to the CHR model, we changed the temperature over the region of formation of the oxygen lines in steps of $100 \mathrm{~K}$ extending $\pm 500 \mathrm{~K}$ from the model. In order to be acceptable, the H $\alpha$ and $\mathrm{H} \beta$ lines are well replicated (their equivalent widths changed by only $\sim 2 \%$ ). The equivalent widths of the oxygen lines changed by an average of $9 \%$ for these models suggesting that the strength of the oxygen lines depends principally on the abundance of oxygen. If Ly- $\beta$ pumping is omitted, the equivalent widths of the triplet decrease by $\sim 20 \%$. In conclusion, the model is reasonably fixed by the hydrogen lines and changing the temperature where the oxygen lines are formed creates a negligible difference in the equivalent widths of the oxygen lines. 
The calculated profiles for 3 values of the O abundance (Fig. 4) are compared to observations of the Omega Cen giants, LEID 54064 and LEID 54084. Equivalent widths from the models are given in Table 1. The spectra from Magellan/MIKE have S/N reaching 100-200, but the oxygen lines are weak, and the forbidden lines are challenged by blending with other atomic lines, telluric lines $(\lambda 6300)$, and a continuum marred by a broad autoionizing line of Ca I ( $\lambda 6362)$. However, the observations of both forbidden transitions and the triplet indicate generally consistent agreement with model profiles having similar values of the oxygen abundance. Synthesis of the $\lambda 6300$ transition (Johnson and Pilachowski 2010) for LEID 54064 and LEID 54084 suggests $\log \mathrm{O} / \mathrm{H}=7.53$ and 7.63 respectively where $\log \mathrm{H}=12.00$. From our CHR model, the $\lambda 7775$ multiplet yields $\log \mathrm{O} / \mathrm{H}=7.04$ in LEID 54064, a reduction of $\sim 0.5$ dex; the forbidden transitions and the $\lambda 7775$ multiplet in LEID 54084 also appear consistent with a low abundance: $\sim 7.1$, a reduction also by $\sim 0.5$ dex. These CHR models eliminate the abundance differences found (Takeda 2003) between forbidden and triplet lines using RCE/NLTE models. The abundance changes suggested here by the 1D CHR/NLTE model are larger for both the forbidden and triplet transitions than those deduced from 3D/NLTE photospheric models of a subgiant star (Amarsi et al. 2015) which indicate essentially no change in the abundance from the forbidden lines when compared to 1D/LTE models and a more modest correction $(\sim-0.14 \mathrm{dex})$ for the triplet.

\section{DISCUSSION AND CONCLUSIONS}

Adoption of a stellar atmospheric model with a chromosphere, in a 1D non-LTE calculation of level populations and line strengths for O I, including photoexcitation by Ly $\beta$ affects both the forbidden transitions (6300 $\AA$ and $6363 \AA)$ as well as the triplet transition (7771-7775 $)$. These transitions are systematically changed as compared to a RCE model: a chromosphere strengthens both the forbidden transitions and the triplet transitions. With the (slightly) increased temperature in the CHR model, higher levels of O I are populated.

These results challenge the often cited statement (Kiselman 2001) that the forbidden lines ( $\lambda 6300$ and $\lambda 6363)$ can be analyzed with an LTE approximation. While this may be true for a photospheric model, the presence of a chromosphere increases the forbidden lines substantially. Thus "LTE abundances" will overestimate the true oxygen abundance by a factor of $\sim 3$ in these stars.

Our results suggest that the inferred oxygen abundance is decreased when a CHR model is invoked. Since differing $\mathrm{H}$-alpha profiles and variation in $\mathrm{H}$-alpha profiles in red giants suggest intrinsic changes in the dynamics, and most likely the temperature and density structure of the stellar atmosphere, it is reasonable to expect that such changes signal different chromospheric structures and the strengths of the oxygen lines may also differ between similar metalpoor red giants. Of course there is no reason to believe that the absolute abundance of $\mathrm{O}$ changes due to chromospheric variability in a given star. Chromospheres in metal-poor field stars are anomalously 'strong' considering the lower metal abundance of these objects, and the chromospheric radiative losses do not appear to scale with metallicity (Dupree et al. 2007). Since chromospheric structures can differ, we conjecture that the 'correction' for the oxygen abundance may not have a constant value, even for similar stars. Thus one might expect that a Na/O anticorrelation may 'spread out' due to chromospheric variation. Further calculations are necessary to explore these effects. The distribution could become bimodal or multimodal much as photometry reveals multiple populations in clusters.

Metal-poor giants in the field do not display the typical Na-O anti-correlation found in globular cluster giants (Kraft 1994). A possible decrease in the oxygen abundance could suggest (Gratton et al. 2000) that deep mixing episodes occur on the upper red giant branch to bring processed material from the CNO cycle to the surface; alternatively, abundance patterns might be attibuted to the initial composition of the star-forming material from a previous generation of more massive stars.

The effects of non-LTE and chromospheres have been considered for dwarf stars of solar metallicity. Maderak et al. (2013) concludes that oxygen abundances derived from the triplet using photospheric models, if corrected for nonLTE, may be reliable for solar-type dwarf stars with solar metallicities. Recent calculations of the triplet for a solar model including a chromosphere suggest less than $1.5 \%$ difference in the equivalent widths for a non-LTE calculation (Dobrovolskas et al. 2014). However, it is not stated whether Lyman- $\beta$ pumping was included, and the case of low metallicities remains to be considered.

Multiple populations in globular clusters and their origin are also assessed in part by oxygen abundances. A decrease in the $\mathrm{O} / \mathrm{H}$ abundance of $\sim 0.5 \mathrm{dex}$, has the potential to affect many abundance relationships (cf. Fig. 3, Gratton et al. 2012) and in particular the important $\mathrm{Na} / \mathrm{O}$ anti-correlations. If all oxygen abundances in cluster giants were to be uniformly reduced by 0.5 dex, this would imply that the oxygen yield from Type II SN (Nomoto et al. 2006) is overestimated and cannot satisfy the abundance patterns. An increase in the contribution of AGB stars of $\sim 5 \mathrm{M}_{\odot}$ would appear to be necessary (Johnson and Pilachowski 2010). Our two target stars have similar oxygen abundances, but different values of $\mathrm{Na} / \mathrm{Fe}$, thus challenging the $\mathrm{Na}-\mathrm{O}$ anti-correlations. Sodium is enhanced in the 
He-rich star (LEID 54084). Johnson and Pilachowski (2010) found $[\mathrm{Na} / \mathrm{Fe}]=+0.25$ (LEID 54084) and $[\mathrm{Na} / \mathrm{Fe}]=+0.16$ (LEID 54064). Another determination (Dupree et al. 2011) for the same stars, yielded [Na/Fe] $=+0.37$ (LEID 54084) and $[\mathrm{Na} / \mathrm{Fe}]=-0.14$ (LEID 54064). The sense of the variation agrees between both determinations, but the difference is larger from the later result. The typical abundance pattern suggests that enhanced He accompanies enhanced Na, and enhanced $\mathrm{Na}$ should correlate with a lower O abundance (Gratton et al. 2012) which it does not in these two target stars. Abundance patterns in the two target stars are consistent with respect to He and $\mathrm{Na}$, but display similar lowered oxygen abundances. However a note of caution emerges in that the radial distribution of the abundances in Omega Centauri differs between oxygen and sodium (Johnson \& Pilachowski 2010) suggesting that a clean anti-correlation may actually not exist.

Accurate abundance values may shed light on the discreteness of photometry of globular clusters versus the continuity of abundance determinations (Milone et al. 2015; Renzini et al. 2015). A wider range of CHR models can address the precise abundances necessary for understanding the hosts of exoplanets (Bedell et al. 2014; Teske et al. 2014). Other elements merit analysis with realistic chromospheric models too such as Fe I, Fe II, Na I, and Mg I. To follow up these results, a broader array of models must be evaluated. At present, we cannot assume the structure of a stellar chromosphere a priori, but it must be constrained by spectroscopic observations of the transitions typical of chromospheric temperatures: $\mathrm{He}$ I, Ca II, $\mathrm{H} \alpha, \mathrm{Na}$ D, Mg II etc. A confounding parameter could be activity variations: the chromosphere itself changes structure and the impact on the oxygen line strengths needs to be assessed. Thus differential abundances could be suspect. If a chromosphere results from magnetic dynamo activity, one might suggest that older, metal-poor stars would experience a decay in activity. However, a temperature rise could result from pulsations and shock formation. In luminous metal-poor stars, the $\mathrm{H} \alpha$ line emission varies in strength and asymmetry (cf. Mészáros et al. 2009), most likely from modest pulsations. In addition, weak magnetic fields detected in luminous stars (cf. Landstreet 2015) could be present in the oldest stars and cause heating (Musielak et al. 2002). In any case, with a good understanding of the chromospheric structure, the oxygen line strengths and others can be calculated with confidence.

We appreciate the thoughtful comments of Christian Johnson on this research.

Facility: Magellan:Clay (MIKE)

\section{REFERENCES}

Amarsi, A. M., Asplund, M., Collet, R., \& Leenaarts, J. 2015, MNRAS, 454, L11

Amarsi, A. M., Asplund, M., Collet, R., \& Leenaarts, J. 2016, MNRAS, 455, 3735

Asplund, M. 2005, ARA\&A, 43, 481

Asplund, M., Grevesse, N., Sauval, A. J., \& Scott, P. 2009, ARA\&A, 47, 481

Asplund, M., Nordlund, Å., Trampedach, R., \& Stein, R. F. 1999, A\&A, 346, L17

Avrett, E. H., \& Loeser, R. 2008, ApJS, 175, 229

Ayres, T.R., Fleming, T. A., Simon, T. et al. 1995, ApJS, 96, 223

Badnell, N. R., O'Mullane, M. G., Summers, H. P. et al. 2003, A\&A, 406, 1151

Barklem, P. S. 2007, A\&A, 462, 781

Bedell, M., Meléndez, J., Bean, J. et al. 2014, ApJ, 795, 23

Boesgaard, A. M., Lum, M. G., \& Deliyannis, C. P. 2015, ApJ, 799, 202

Bowen, I. S. 1947, PASP, 59, 196

Castelli, F., \& Kurucz, R. L. 2004, arXiv:0405087 (see http://kurucz.harvard.edu/)

Dobrovolskas, V., Kučinskas, A., Bonifacio, P. et al. 2014, A\&A, 565, A121

Dupree, A. K., Hartmann, L., \& Avrett, E. H. 1984, ApJL, 281, L37

Dupree, A. K., Li, T. Q., \& Smith, G. H. 2007, AJ, 134, 1348

Dupree, A. K. \& Smith, G. H. 1995, AJ, 110, 405

Dupree, A. K., Strader, J., \& Smith, G. H. 2011, ApJ, 728, 155

Drawin, H. W. 1969, Z. Physik, 225, 483

Fabbian, D., Asplund, M., Barklem, P. S., Carlsson, M., \& Kiselman, D. 2009, A\&A, 500, 1221
Frebel, A., \& Norris, J. E. 2015, ARA\&A, 53, 631

Freytag, B., Allard, F., Ludwig, H.-G., Homeier, D., \& Steffen, M. 2010, A\&A 513, A19

Fulbright, J. P., \& Johnson, J. A. 2003, ApJ, 595, 1154

Gratton, R. G., Carretta, E., Bragaglia, A. 2012, A\&ARv., 20, 50

Gratton, R. G., Carretta, E., Eriksson, K., \& Gustafsson, B. 1999, A\&A, 350, 955

Gratton, R. G., Lucatello, S., Sollima, A. et al. 2015, A\&A, 573, A92

Gratton, R. G., Sneden, C., Carretta, E., \& Bragaglia, A. 2000, A\&A, 354, 169

Gustafsson, B., Edvardsson, B., Eriksson, K. et al. 2008, A\&A, 486, 951

Haisch, B. M., Linsky, J. L., Weinstein, A., \& Shine, R. A. 1977, ApJ, 214, 785

Hauschildt, P. H., Allard, F., \& Baron, E. 1999, ApJ, 512, 377

Israelian, G., Shchukina, N., Rebolo, R. et al. 2004, A\&A, 419, 1095

Johnson, C. I., McWilliam, A.,C., \& Rich, R. M. 2013, ApJ, 775, L27

Johnson, C. I., \& Pilachowski, C. A. 2010, ApJ, 722, 1373

Johnson, C. I., \& Pilachowski, C. A. 2012, ApJ, 754, L38

Kaulakys, B. 1985, J. Phys. B., 18, L167

Kiselman, D. 2001, NewAR, 45, 559

Korotin, S. A., Andrievsky, S. M., Luck, R. E. et al. 2014, MNRAS, 444, 3301

Kraft, R. P. 1994, PASP, 106, 553

Landstreet, J. D. 2015, Proc. IAU, 305, 12

Maderak, R. M., Deliyannis, C. P., Anthony-Twarog, B. J. et al. 2015, AJ, 149, 141 


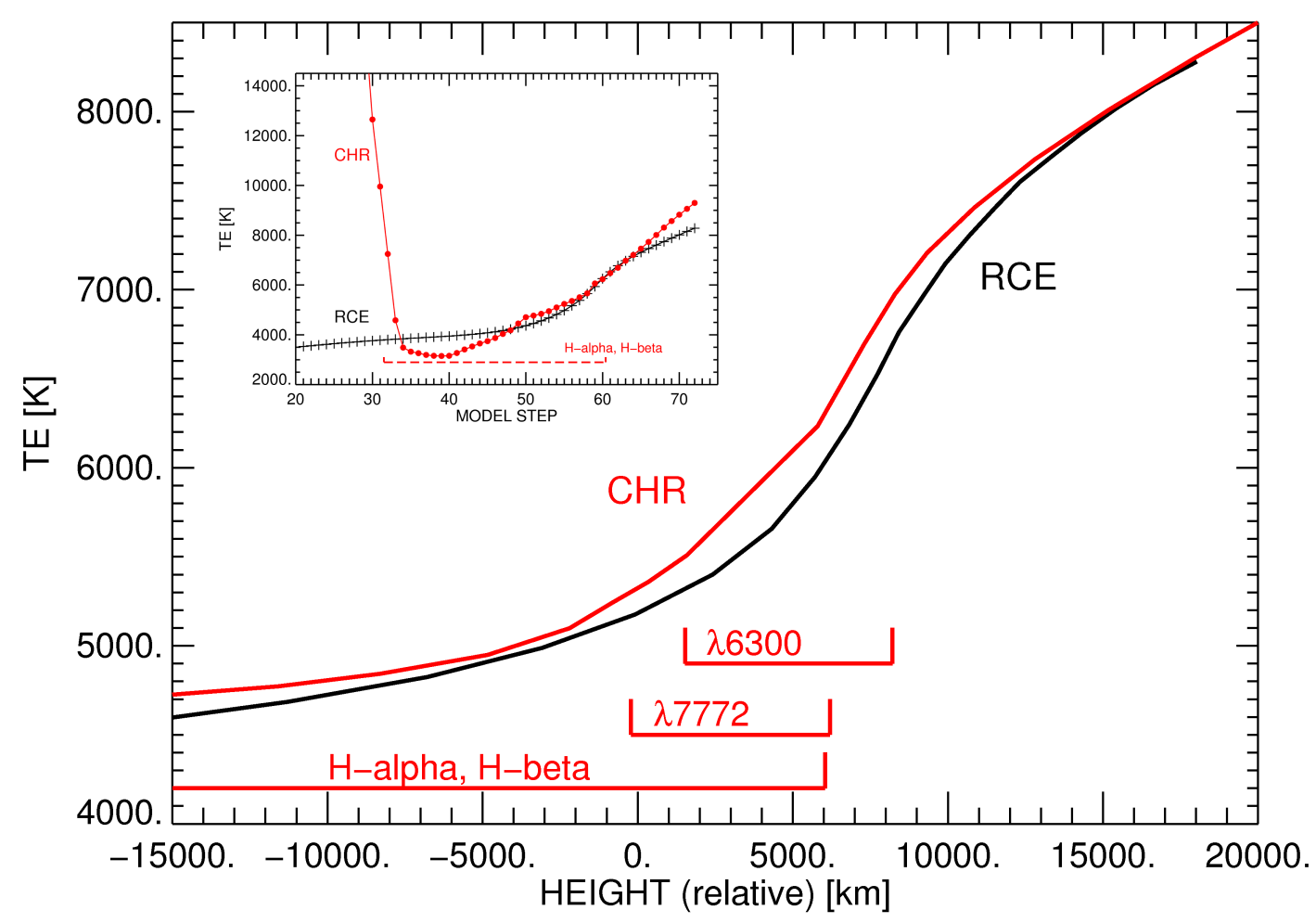

Figure 1. Region of formation of the $\mathrm{O}$ I lines and $\mathrm{H} \alpha$ and $\mathrm{H} \beta$ in the CHR model. This region is defined by the full width at half maximum of each line contribution function for a disk-centered profile taken at line center and $\pm 1 \AA$ from line center. The $\mathrm{RCE}$ model has been moved outward in height for illustration. In the CHR model, Height $=0$ corresponds to $\tau_{5000}=1$. The CHR model extends much beyond the $\mathrm{RCE}$ model to $\sim 1.8 \mathrm{R}_{\star}$.

Marino, A. F., Milone, A. P., Sneden, C. et al., 2012a, A\&A, $541, \mathrm{~A} 15$

Marino, A. F., Milone, A. P., Piotto, G. et al., 2012b, ApJ, 746, 14

Martínez-Arnáiz, Maldonado, J., Montes, D., Eiroa, C., \& Montesinos, B. 2010, A\&A, 520, A79

Mészáros, Sz., Avrett, E. H., \& Dupree, A. K. 2009, AJ, 138, 615

Mészáros, Sz., Allende Prieto, C., Edvardsson, B., et al. 2012, AJ, 144, 120

Milone, A. P., Marino, A. F., Piotto, G., et al., 2015, ApJ, 808, 51

Moos, H. W., \& Rottman, G. J. 1972, ApJL, 174, L73

Musielak, Z. E., Rosner, R., \& Ulmschneider, P. 2002, ApJ, 573, 418

Nomoto, K., Tominaga, N., Umeda, H., Kobayashi, C., \& Maeda, K. 2006, Nucl. Phys. A, 777, 424
Renzini, A., D'Antona, F., Cassisi, S. et al. 2015, MNRAS, 454, 4197

Schuler, S. C., King, J. R, Terndrup, D. M. et al. 2006, ApJ, 636, 432

Seaton, M. J. 1962, PPS, 79, 1105

Shchukina, N. G., Trujillo Bueno, J., \& Asplund, M. 2005, ApJ, 618,939

Takeda, Y. 1997, PASJ, 49, 471

Takeda, Y. 2003, A\&A, 402, 343

Teske, J. K., Cunha, K., Smith, V. V., Schuler, S. C., \& Griffith, C. A. 2014, ApJ, 788, 39

VandenBerg, D. A., Bond, H. E., Nelan, E. P. et al. 2014, ApJ, 792,110

Wheeler, J. C., Sneden, C., \& Truran, J. W. 1989, ARA\&A, 27, 279 

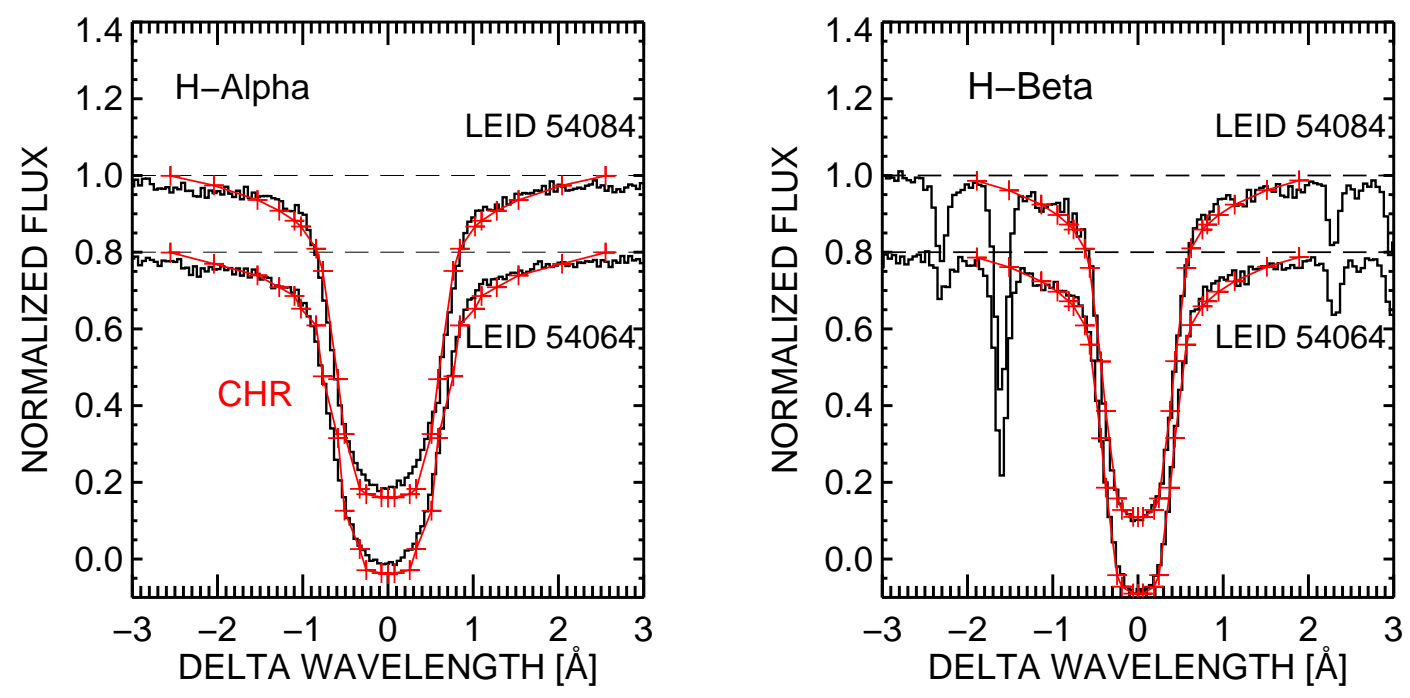

Figure 2. $\mathrm{H} \alpha$ and $\mathrm{H} \beta$ profiles as observed (Dupree et al. 2011) in two Omega Cen giants, LEID 54084 and LEID 54064 (black curves), and the calculated profiles using the same chromospheric model (red curves). The profiles for LEID 54064 are offset by -0.2 for display.

Table 1. Equivalent Widths (mA) and Abundances from Atmospheric Models

\begin{tabular}{lcccccc}
\hline \hline Model & $(\mathrm{O} / \mathrm{H})$ & $\lambda 6300$ & $\lambda 6363$ & $\lambda 7772$ & $\lambda 7774$ & $\lambda 7775$ \\
$\begin{array}{c}\text { E.P.(eV) } \\
\text { log gf }\end{array}$ & Model & 0.0 & 0.019 & 9.15 & 9.15 & 9.15 \\
$\mathrm{RCE}$ & & -9.78 & -10.26 & 0.36 & 0.21 & -0.02 \\
$\mathrm{CHR}$ & 7.64 & 17.5 & 5.7 & 13.5 & 10.5 & 7.0 \\
$\mathrm{CHR}$ & 7.64 & 28.7 & 10.7 & 57.9 & 42.9 & 27.7 \\
$\mathrm{CHR}$ & 7.34 & 14.2 & 5.3 & 31.1 & 23.2 & 14.5 \\
$\mathrm{O} / \mathrm{H}$ Abundance & 7.04 & 7.4 & 2.5 & 16.8 & 12.3 & 7.4 \\
$\mathrm{RCE}{ }^{1}$ (LEID 54064) & & 7.53 & $\ldots$ & $\ldots$ & $\ldots$ & $\ldots$ \\
$\mathrm{RCE}^{1}$ (LEID 54084) & & 7.63 & $\ldots$ & $\ldots$ & $\ldots$ & $\ldots$ \\
$\mathrm{CHR}^{2}$ (LEID 54064) & & $\ldots$ & $\ldots$ & 7.04 & 7.04 & 7.04 \\
$\mathrm{CHR}^{2}$ (LEID 54084) & & 7.1 & 7.1 & $\ldots$ & 7.04 & 7.04 \\
\hline
\end{tabular}

$1^{1}$ Johnson \& Pilachowski 2010

2 This paper. 

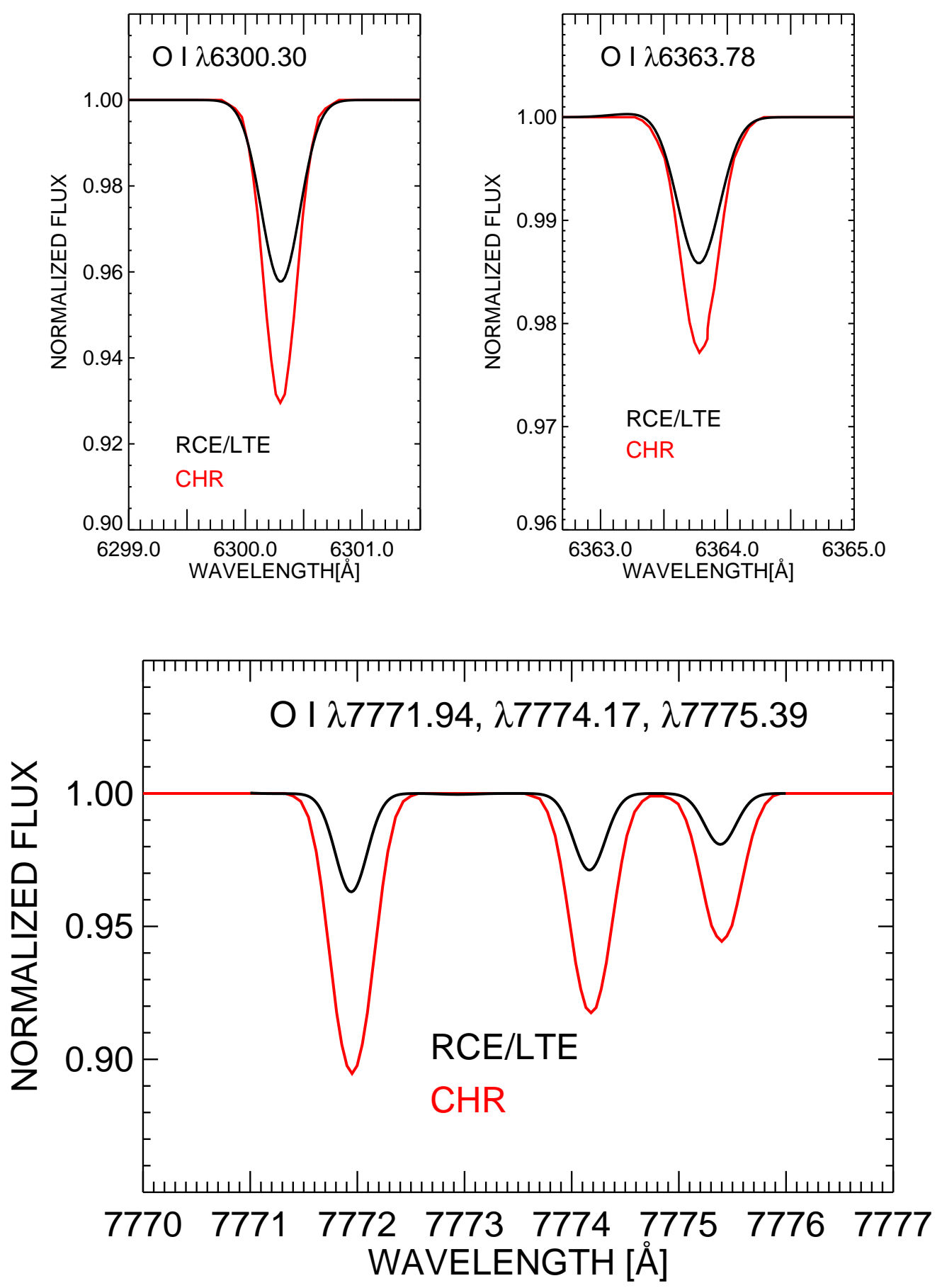

Figure 3. Oxygen profiles for a RCE and CHR model. These are calculated for $[\mathrm{Fe} / \mathrm{H}]=-1.7$, and are alpha enhanced; Oxygen abundance set to 7.64. All transitions are calculated (radiative). The CHR models produce stronger lines than RCE models in all transitions. 

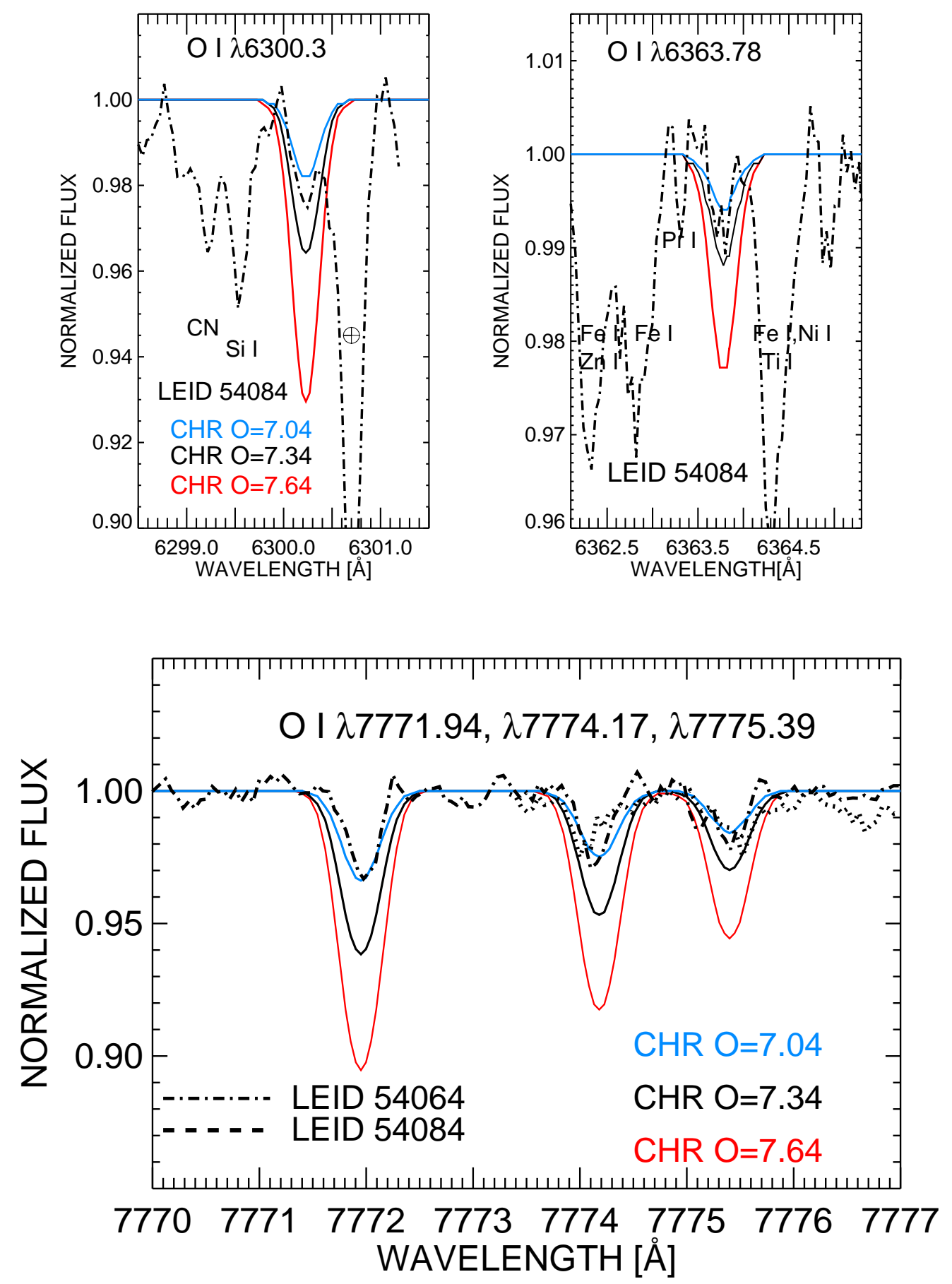

Figure 4. Calculated oxygen profiles with a CHR model for various values of the oxygen abundance, log $\mathrm{O} / \mathrm{H}$ on a scale where $\log \mathrm{H}=12.0$. Observed profiles with Magellan/MIKE (Dupree et al. 2011) for the target stars, LEID 54064 and LEID 54084 are shown. 